\title{
Spatial inhomogeneities in the energy response of a tunnel junction detector due to penetration of Abrikosov vortices
}

\author{
J.B. le Grand ${ }^{\mathrm{a}, *, 1}$, J. Martin ${ }^{\mathrm{a}}$, R. Gross ${ }^{\mathrm{a}}$, R.P. Huebener ${ }^{\mathrm{a}}$, A.W. Hamster ${ }^{\mathrm{b}}$, G.C.S. Brons ${ }^{\mathrm{b}}$, \\ D.J. Adelerhof ${ }^{b}$, J. Flokstra ${ }^{b}$, P.A.J. de Korte ${ }^{c}$ \\ "Physikalisches Institut, Lehrstuhl Experimentalphysik 11. University of Tübingen, Morgenstelle 14, D-72076, Tübingen. Germany \\ "University of Twente. Department of Applied Physic's, P.O. Box 217, 7500 AE Enschede, Netherlands \\ 'Space Research Organization Netherlands. Sorbonnelaan 2, 3584 CA Utrecht, Netherlands
}

\begin{abstract}
Ahstract
For the application of superconductive tunnel junctions (STJs) as high resolution X-ray detectors the homogeneity of the detector response is of utmost importance. In this article it is shown how this homogeneity is degraded by the penetration of Abrikosov vortices (AVs) into the junction electrodes. These AVs may exist because of small misalignments in the parallel magnetic field, which is applied to suppress the Josephson current and Fiske steps. By means of Low Temperature Scanning Electron Microscopy (LTSEM) the response of the tunnel junction to local energy deposition is mapped and found to be reduced in areas where AVs are present. On the basis of these experiments the threshold perpendicular field at which vortices start to penetrate the junction is derived.
\end{abstract}

\section{Experiments}

In this experiment a $\mathrm{Nb} / \mathrm{Al} / \mathrm{AlO} \mathrm{v}_{\mathrm{v}} / \mathrm{Al} / \mathrm{Nb}$ tunnel junction detector with a barrier size of $100 \times 100 \mu \mathrm{m}^{2}$ and a current density of $500 \mathrm{~A} / \mathrm{cm}^{2}$ was studied by LTSEM. The junction was scanned with a beam of $20 \mathrm{keV}$ electrons. The absorption of the energy of an electron pulse is equivalent to the absorption of an X-ray photon. A parallel magnetic field was applied during the whole experiment to suppress the Josephson current and Fiske resonances. By means of a second coil, a perpendicular magnetic field could be applied.

In Fig. 1a, the detector response is shown without perpendicular magnetic field. On the left, an area of $30 \times$ $30 \mu \mathrm{m}^{2}$ of reduced signal is observed. In this area the counter electrode is covered by the contacting lead. Also towards the edges the signal is slightly reduced, which is attributed to quasiparticle loss processes near the edges. When applying a perpendicular magnetic field of up to $13 \mathrm{G}$, no change in the $I / V$ characteristic and LTSEM signal of the junction was detected. At about $13 \mathrm{G}$, magnetic field flux started to penetrate the junction. The lower critical perpendicular field $H_{c 1, \text { quare }}$ of a square superconductor with length $L$ and thickness $d$ is given by

\footnotetext{
* Corresponding author. Tel. +497071296324, fax +497071 296322, e-mail legrand@brahms.pit.physik.uni-tuebingen.de.

'Working as an external ESA research fellow.
}

$$
H_{\mathfrak{c}^{\prime}, \ldots q u a r e}=\frac{2 d}{L} H_{\mathrm{c}^{\prime} 1}
$$

with $H_{\mathrm{cl}}$ the lower critical field of bulk material. For our junction $L=100 \mu \mathrm{m}, \quad d=500 \mathrm{~nm}$ and $H_{c 1 . .4 u \text { uare }}=$ $13 \pm 1 \mathrm{G}$, so we derive $H_{\mathrm{c} 1}=1300 \pm 100 \mathrm{G}$, which is in agreement with $H_{\mathrm{c},}$ of niobium [1]. Due to flux creep, the stabilization of the flux penetration took about 10 minutes, during which the quasiparticle tunnel current, the Josephson current and the detector response were very unstable. After stabilization scan $1 \mathrm{~b}$ was made. It is shown that the signal is reduced at the edges of the junction, but not in the corners. The reduction of the signal is due to quasiparticle loss in AVs. The distribucion of penetrated flux at the edges and not in the corners is in agreement with magnetooptical measurements on flux distribution [2].

After applying a very strong perpendicular field for some minutes and then removing the perpendicular field completely, scan Ic was made. It is assumed that in the strong field, flux penetrated the junction everywhere. After removing the field. flux is expelled from the edges of the junction, resulting in a flux gradient from the edges towards the middle of the electrode, as is derived from the intensity distribution of Fig. Ic.

Next, we scanned the junction with a very strong electron beam without applied perpendicular field. The scan made after this procedure reproduced the first figure, indicating that this procedure removes all penetrated flux. Finally, a perpendicular field of $6 \mathrm{G}$ was applied to that junction. This field is not large enough to drive flux into 


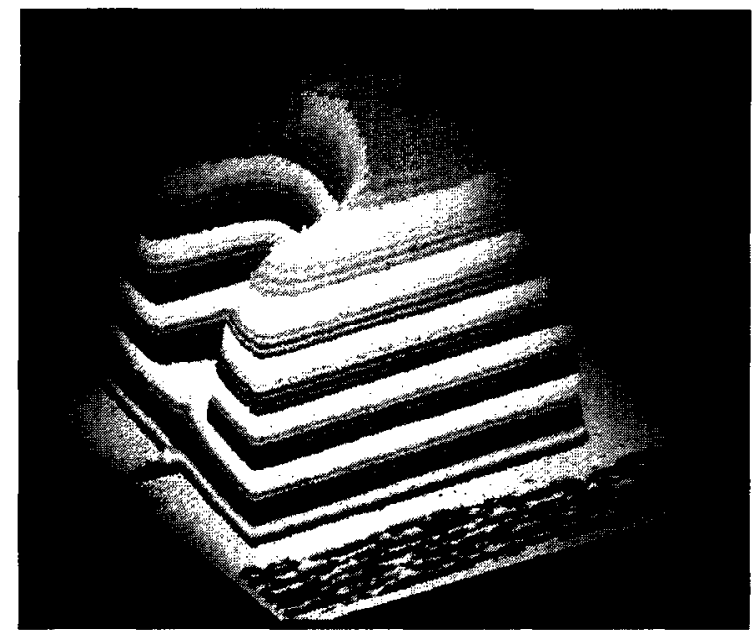

(a)

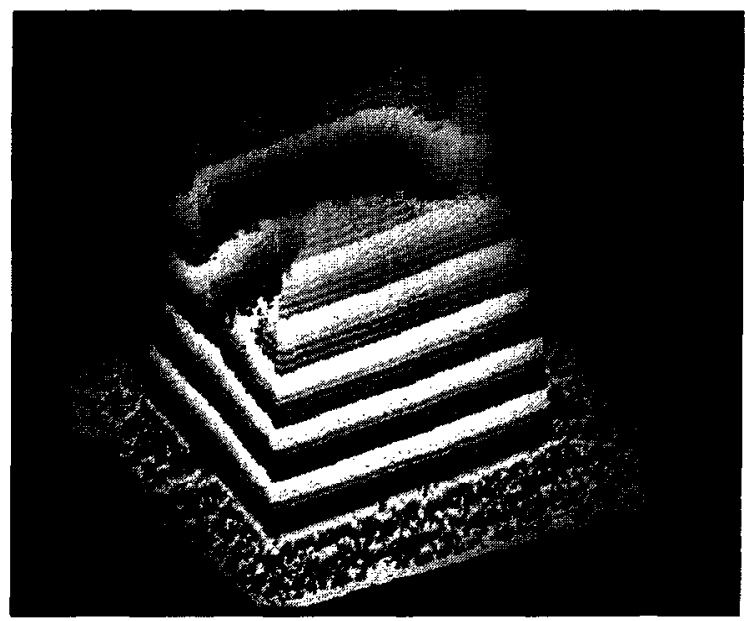

(c)

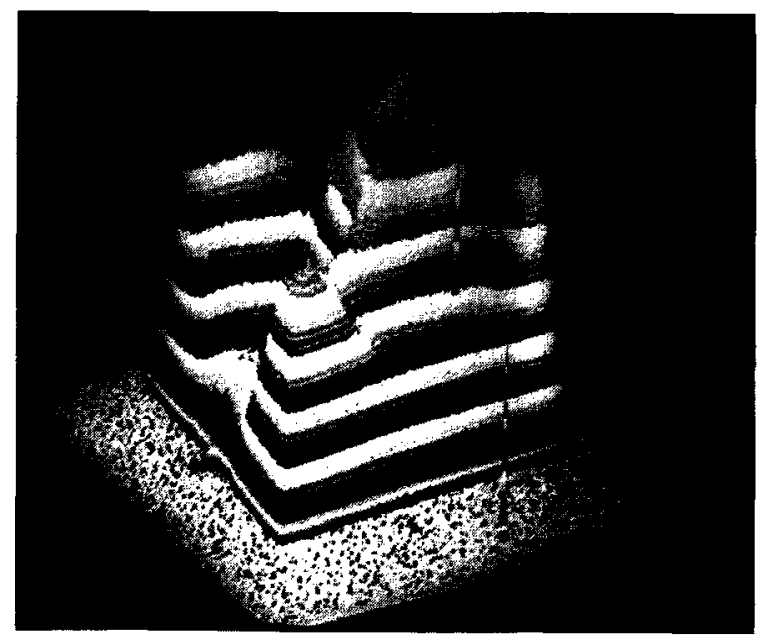

(b)

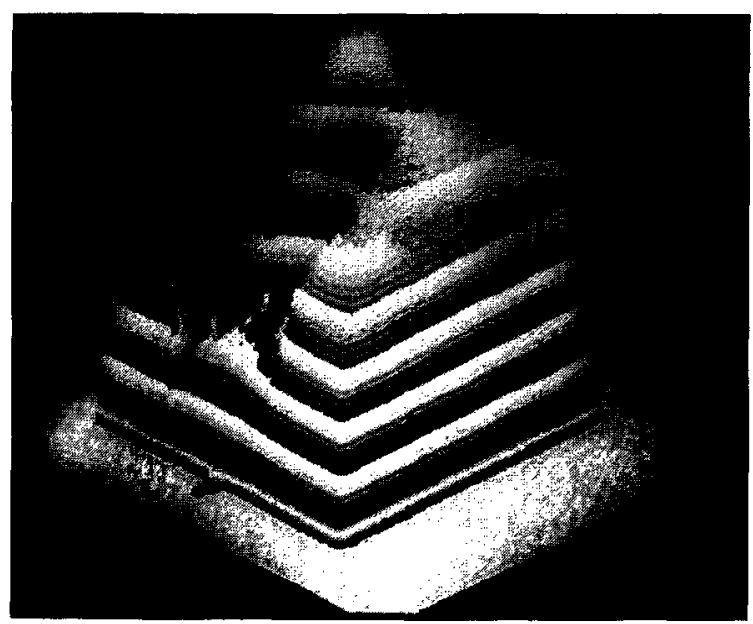

(d)

Fig. 1. LTSEM scans, showing various distributions of flux vortices. The vertical axis corresponds to the signal height.

the junction. Now, a small window on the left corner of the junction was irradiated with a very strong electron beam. The beam current was a factor $10^{5}$ larger than during the mapping. After this procedure we obtain the detector response shown in Fig. 1d. It is shown that at the position were the strong irradiation warmed up the junction, flux could enter the junction even for $6 \mathrm{G}$. The signal in this area is reduced.

\section{Conclusions}

In areas where flux penetrated the junction the signal is reduced. The distribution of $\mathrm{AVs}$ is a function of the magnetic history of the sample. The various distributions observed are in agreement with expectations. The spontaneous penetration of flux starts at $13 \mathrm{G}$, which is agreement with a bulk critical magnetic field of $1300 \mathrm{G}$. By applying strong electron radiation under conditions of a weak magnetic field, flux penetration can be stimulated locally.

\section{References}

[1] T. McConville and B. Serin, Phys. Rev. 140 (1965) All69.

[2] V.A. Rowe. R.P. Huebener and R.T. Kampwirth, Phys. Status Solidi A 4 (1971) 513 . 